\title{
The PASS Effect
}

\section{How Peer Assisted Study Sessions Contribute to a Computing Community}

\author{
Lesley May \\ School of Physical Sciences and \\ Computing \\ University of Central Lancashire \\ Preston, UK \\ LMay@uclan.ac.uk
}

\author{
Nicky Danino \\ School of Physical Sciences and \\ Computing \\ University of Central Lancashire \\ Preston, UK \\ NDanino@uclan.ac.uk
}

\begin{abstract}
Computing at UCLan introduced Peer Assisted Study Sessions (PASS) across all their courses in 2016, a scheme which has been identified as 'Best Practice' by the British Computer Society. Pairs of higher-year students (PASS Leaders) facilitate a group of lower year students to discuss and resolve problems during informal weekly sessions which are, in addition to the normal teaching events, scheduled sympathetically with the students' timetables.

The sessions offer students extra support, tailored to their needs by identifying topics that they would like to explore further. The PASS Leaders facilitate group discussions or exercises designed to enhance the students' understanding of those topics in engaging and fun sessions. The participants benefit by having a greater understanding of the topics, enhancing their friendship with their peers and Leaders, providing a wider informal support network. Moreover, because of discussions with higher year students, attendees have a greater understanding of what their course is about and where topics fit into the wider context of their degree. There is a weekly debrief of PASS Leaders with a member of staff (PASS Supervisor) to discuss issues raised and offer ongoing support to the PASS Leaders. Troublesome topics are reported to the teaching team, so they can be addressed for future classes. The PASS Leaders benefit as much from the scheme as the students they support. Leaders say that participating in PASS has increased their confidence and has improved their organisational and interpersonal skills, amongst other things. Revisiting topics from previous years can also enhance their understanding of the material. From an academic perspective, the scheme is working well, embedding into the culture of student life. It has fostered the creation of a community across all courses and years, which increases inclusivity and brings a greater sense of belonging to our student body.
\end{abstract}

Permission to make digital or hard copies of all or part of this work for personal or classroom use is granted without fee provided that copies are not made or distributed for profit or commercial advantage and that copies bear this notice and the full citation on the first page. Copyrights for components of this work owned by others than ACM must be honored. Abstracting with credit is permitted. To copy otherwise, or republish, to post on servers or to redistribute to lists, requires prior specific permission and/or a fee. Request permissions from

Permissions@acm.org.

CEP 2020, January 9, 2020, Durham, United Kingdom

(C) 2020 Association for Computing Machinery.

ACM ISBN 978-1-4503-7729-4/20/01 ..\$15.00

https://doi.org/10.1145/3372356.3372371

\section{CCS CONCEPTS}

- Applied Computing $\rightarrow$ Education $\rightarrow$ Collaborative Learning

\section{KEYWORDS}

Student experience, student partnership, student peer support.

ACM Reference format:

Lesley May and Nicky Danino. 2020. The PASS Effect: How Peer Assisted Study Sessions Contribute to a Computing Community. In Computing Education Practice (CEP '19), January 9, 2020, Durham, United Kingdom. ACM, New York, NY, USA, Article 1, 4 pages.

\section{Introduction}

This paper depicts a novel and integrated approach to supporting undergraduate students in Computing at UCLan (University of Central Lancashire) via the introduction of PASS (Peer Assisted Study Sessions), a scheme which has been identified as 'Best Practice' by the British Computer Society. This approach is intended to be both academic and pastoral in nature, with the aim of increasing student engagement both in their studies, and in the Computing community at the university.

Heaton-Shrestha et al. [2] propose that the most influential model developed to account for the early departure of students from HE is that of Tinto [8]. Tinto states that the student resolution to continue or withdraw is strictly linked to the degree to which he or she has achieved in becoming both socially and academically amalgamated into the institution. Forbes [1] adapted this model, further considering the needs of part-time students, and including outside influences such as the necessity for earning money. This 'new retention model' highlights the significance of peer interaction to support retention. Forbes also highlights the importance of academic and social adjustment, and of suitable and correct information being given to prospective students before enrolment. A study carried out by Hodgson, May, \& Marks-Maran [3] discovered that peer support and social engagement were vital in retaining students in the first year of a course.

Given that education has been experiencing a paradigm change away from teaching-as-instruction towards student-centred learning [7], educators must re-evaluate both the approach of their delivery and their method of assessment. Therefore, the curriculum has been planned more and more around learning outcomes as 
opposed to content [5]. Kolb [4] highlights the need for identifying different learning styles in students. He advocates that an individual gains knowledge through assuming a task. They then are required to reflect on their involvement and try to fathom the experience through enquiry and conceptualisation. The individual then makes choices based on what they have learned, decides on their next action, and undertakes another task. Learning is consequently cyclical and certainly never reaches completion. The practice is continuously recurring.

Our approach is centred around creating a positive environment for the students who are attending PASS, as much as it is about fostering a scheme that also benefits the students who are providing the peer support.

\section{Background}

Computing at UCLan is a medium sized department by UK standards: 23 academic staff teach on a variety of three and four year undergraduate degrees and different taught and research postgraduate degrees, to which approximately 200 undergraduate and 300 postgraduate students are admitted each year. In addition, we also have approximately 40 foundation year students every year, several cohorts of apprentices that are integrated within the undergraduate programme, and a yearly intake of apprentices that are taught once a month for a week at a time.

UCLan is a widening participation university and undergraduate Computing students come from all manner of academic backgrounds. Many have studied either Computing or IT at school or college, whilst others have not undertaken any formal qualifications in the subject. Computing is run as an almost common first year, with entry requirements of up to 112 UCAS tariff points at A2 or BTEC National Diploma MMM-DMM and 5 GCSEs at grade $\mathrm{C}$ or above including Maths and English. Students study the first year to gain a grounding foundation in Computing, and then progress to year 2 where they choose a specialism. Specialisms range from Computer Games Development, Computer Networks and Security, Forensic Computing and Security, Computer Science, Software Engineering, Cyber Security, and Computing, which is a student self-select course that offers a more flexible programme of study.

As the profile of our Computing department shows, generally, our staff/student ratio has always been high, so we are always on the lookout for practical ways to support students that do not drastically increase workload for academic staff. Before we introduced PASS, in addition to the standard timetabled hours, staff were all holding additional support sessions to help students who were struggling with basic concepts, with little success. The additional support sessions were a strain on staff, not well attended by students, and often a repeat of what had already been covered during the timetabled hours. Retention in Computing subjects is an issue for many universities, including UCLan, so ways of engaging students and creating a sense of belonging were explored, with PASS deemed a viable option.

We decided to introduce PASS across all Computing undergraduate courses in 2016 after one of our academics (Lesley
May) attended a PASS Supervisor training session run by the European Centre for SI-PASS.

\section{The PASS Scheme}

\subsection{What is PASS?}

PASS focuses on creating a supportive environment, empowering greater conceptual understanding of essential academic principles and increasing individual confidence rather than superficial strategic learning to pass coursework and exams. PASS supports:

○ Academic Performance: Conceptual understanding of academic material

- Learning Strategies: Practical approaches to study

- Orientation and Transition: Adaptation and socialisation.

PASS utilises the experience and expertise of higher year students (PASS Leaders) by training them as facilitators, not teachers or tutors. The Leaders support their group of lower year students in finding solutions to their problems themselves, through facilitated discussion and guided questioning. The group sessions are aligned to each course of study wherever possible, with both Leaders and attendees studying the same programme. Each group's pair of PASS Leaders encourage active learning in an informal, friendly and fun environment that does not seek to replace any form of interaction between staff and student but to provide another opportunity for all students to enter institutional discourse with their peers. It provides a safe environment for students to discuss ideas, share problems and resolve questions in a setting that supplements the core curriculum. Pass Leaders take biscuits to the sessions, and often arrange social activities with their group attendees, going out for meals, going to the cinema or arranging gaming sessions.

Originally, we purchased comprehensive training for PASS Leaders and Supervisors from the European Centre for SI-PASS. The European Centre delivered introductory workshops ("PASS Demystified") and Supervisor Training. With more experience and the scheme being implemented across the wider university, training is now conducted in-house. Supervisor Training covers all aspects of the programme relating to the implementation, ongoing management and evaluation.

\subsection{How Does PASS Work?}

Pairs of higher-year students (PASS Leaders) facilitate a group of lower year students (around 15-20 in each group) to discuss and solve their problems during informal weekly sessions. The weekly one-hour sessions are in addition to the normal teaching events. We include these sessions on the timetable in a sympathetic way, so they are at a time that is convenient to students, and encourages them to attend, for example, straight after a programming lecture (lecture trap), or in the hour following a class in the same building. The sessions offer students extra support, tailored to their needs, because they identify the topics that they would like to explore further. The pair of PASS Leaders working with that group then facilitate group discussions or exercises designed to enhance the 
students' understanding of those topics in an engaging and fun session.

PASS Leaders partake in full training before they can run sessions. Nevertheless, support throughout the academic year is crucial to the success of the programme. There are weekly Leader debriefs with the PASS Supervisor, that concentrate on both process and content of sessions: reflecting on facilitation skills and techniques; reporting troublesome topics so they can be fed back to the teaching team, and an insight into how the current material delivered within the timetabled classes is being received.

PASS Leaders are very much seen as facilitators, and not teachers, so it is especially vital that the sessions are based around existing information that has already been received by the attendees. This approach safeguards against PASS Leaders teaching new content, and instead, incites students to discuss topics, refining their thoughts about the teaching material. Over and above helping the students amalgamate what they have learned about the subject, PASS Leaders also help with study strategies, imparting advice about what works for them and what does not work. Students tend to pay attention to the PASS Leaders regarding the teaching and learning tips as they see them as peers, and feel that they understand what they are going through.

The scheme is voluntary, so not all students attend. Reasons for non-attendance range from having other commitments outside university to not seeing how the sessions would be of benefit to them. Buy-in from the teaching team is critical in promoting PASS to students, so that more students can see how it can enhance their studies. The benefits for those who participate in the sessions are not only that they have a greater understanding of the topics, they have also enhanced their friendship with their peers and with the higher year students, providing a wider informal support network. Moreover, because of discussions with higher year students, attendees have a greater understanding of what their course is about and where topics fit into the wider context of their degree.

\section{Impact}

In 2017, PASS at UCLan was piloted across ten courses across the university. In 2018 the scheme expanded to cover forty courses, with PASS continually expanding across campus. As the scheme has now expanded further than Computing, university-wide support has been put in place for PASS. This includes a central team situated within the Centre of Excellence in Learning and Teaching. The PASS Team run training, attend debriefs, liaise with staff and create generic resources for sessions covering areas such as referencing, note-taking, managing stress and revision. They coordinate Leaders and manage the PASS process to ensure it runs efficiently and effectively. All Leaders are observed once per semester to provide quality assurance and promote best practice.

The PASS Leaders benefit as much from the scheme as the students they support. Leaders say that participating in PASS has increased their confidence and has improved their organisational and interpersonal skills, amongst other things. Revisiting topics from previous years can also enhance their understanding of the material. In 2016, when PASS in Computing was run for the first time, 24 students were trained as Leaders. This was voluntary, and students put themselves forward for the training and to facilitate sessions in their own time. In subsequent years, we have received funding from the University to run PASS, and Leaders get paid for the training, the sessions, and to attend the weekly Leader debrief. This year we have expanded our provision in Computing to encompass sessions for year 0 , year 1 and year 2 students. We have 20 Leaders in place, with some running more than one session.

As part of our yearly evaluation with our leaders, we collect qualitative data to help us explore how we can improve PASS. Comments from Leaders show what they think of PASS.

The PASS Leaders react positively to questions around deepening their understanding of previously studied material, increasing their confidence, improved team-working skills, as well as their planning, communication and presentation skills. All respondents said that they would recommend being a Leader to other students.

"PASS works because students are on both ends of the programme, those who learned from PASS last year build on the advances and techniques of the year before and pass them along to the next years of students. The cycle repeats and we end up with a breadth of knowledge shared across multiple generations of students, which benefits staff, prospective/current students and the University as a whole."

"PASS is incredible, and it really does work. Not having PASS through foundation year was such a struggle and then having it in first year made uni so much better, learning tips and techniques I would never have learnt without PASS. Not only that but I made friends and a community that helped one another throughout the year. If it wasn't for PASS I don't think I would have done so well in first year allowing me to be prepared, now, in second year and making me want to give back and help others as I was helped."

"The reason I wanted to join PASS is because of what an amazing job Beth and Danny had done with our group. The sessions I attended during the first year really helped me out and I genuinely feel like it helped improve my overall module grades by just going to their sessions. The best thing to me about PASS is the fact it's all peer led so us students get to choose what modules/subjects we go over each session, so when you can attend a session that is directly assisting you with the things you may be struggling with, it definitely makes a difference. I also feel that because the PASS leaders are students themselves and have already studied the same/similar modules, it means you can really relate to what they're saying, and it helps create a great bond between students."

From an academic perspective, the scheme is working well, embedding into the culture of student life. It has fostered the creation of a community across all courses and years, which increases inclusivity and brings a greater sense of belonging to our student body. PASS is proven to improve academic performance and achievement, with our research indicating that regular PASS attendees' grades are on average 10-20\% higher than nonattendees. PASS also provides an additional mechanism for communication and feedback between students and teaching staff, 
where issues or concerns can be raised and addressed. PASS does not replace any normal class contact time and is an additional opportunity to practice the skills that students need to succeed.

\section{Benefits}

The benefits derived from PASS can be seen at different levels. For the university, it helps in providing student support in a costeffective and successful manner. PASS Leaders benefit in a variety of ways, most noticeably in the addition to their employability and transferable skills. According to Ody \& Carey [6], "anecdotal feedback from a range of graduate employers recognises that students who engage in voluntary roles, such as a PASS Leader, develop competencies and transferable skills that increase their employability prospects".

Our regular debriefing sessions have created a space for the PASS Leaders, where they share their experiences, frustrations and successes with their peers. This has helped build confidence for individuals and given the group an identity. It has also provided an opportunity for the PASS Leaders to ask questions of the PASS Supervisor.

Extra support for first year students, driven by them and their needs, facilitated and supported by students who had gone through the same studies in a previous year, has meant that attainments of attendees at PASS has improved.

The creation of a community between the different years of the courses, whereby first years could get a feel for what was to come, and the higher years could reassure the first years where content will be beneficial in future years.

The PASS Leaders have grown in confidence and have a better understanding of earlier material, the university systems and services, and how to get the most out of their time at university.

PASS has been instrumental in creating an environment that has germinated the Computer Games Development Society and the Computer Security Society, which are going from strength to strength.

\section{Costs}

Each Leader undergoes extensive training in how to facilitate sessions, learning techniques in how to include the quieter students whilst also effectively harnessing the more confident students. There is obviously a cost associated with the training, in terms of providing the training itself as well as paying the Leaders for attendance.

Each Leader is provided with a hoodie and a Leader's bag containing the necessary tools for successfully running their sessions (post-it notes, white board markers, etc.) from the central scheme. The Computing Leaders also receive a PASS T-Shirt.

After initial training and outlay, there is also the weekly cost of paying the Leaders. Each Leader is paid for three hours per week; an hour for preparation, an hour for running their session and an hour to attend and participate in the debrief. Where Leaders are running more than one session per week, they receive one extra hour's pay, as the prep and debrief are deemed to be already covered.

Academic staff time in the role of the PASS Supervisor is also a consideration. The weekly debrief is an hour, but the follow up, dealing with the feedback from Leaders, is less predictable.

The PASS Team deal with the administration and coordination of the scheme and currently employ four staff.

\section{Conclusions and Future Work}

PASS has been a positive experience for Computing at UCLan. We have recently expanded the programme to include the sessions for Foundation and Year 2 students. If this is successful we are considering implementing PASS at post-graduate level. With a dedicated and enthusiastic PASS Supervisor, this can be integrated into studies to bring a greater community spirit across course and year boundaries, helping students to make the most of their time both in and out of the classroom.

\section{ACKNOWLEDGMENTS}

We'd like the thank the European Centre for SI-PASS for their excellent training programme, and the University of Central Lancashire for paying our fantastic and enthusiastic PASS Leaders for their hard work.

\section{REFERENCES}

[1] A Forbes. 2008. A New Retention Model. High. Educ. Acad. Annu. Conf. 2008 (2008). Retrieved October 21, 2019 from http://epubs.surrey.ac.uk/819293/

[2] C Heaton-Shrestha, S May, and L Burke. 2009. Student retention in higher education: what role for virtual learning environments? J. Furth. High. Educ. 33, 1 (February 2009), 83-92. DOI:https://doi.org/10.1080/03098770802645189

[3] D Hodgson, S May, and Di Marks-Maran. 2008. Promoting the development of a supportive learning environment through action research from the 'middle out.' Educ. Action Res. 16, 4 (December 2008), 531-544. DOI:https://doi.org/10.1080/09650790802445718

[4] D.A. Kolb. 1984. Experiential learning: Experience as the source of learning and development. Prentice-Hall, Englewood Cliffs, NJ.

[5] B Lin and C Hsieh. 2001. Web-based teaching and learner control: a research review. Comput. Educ. 37, 3-4 (November 2001), 377386. DOI:https://doi.org/10.1016/S0360-1315(01)00060-4

[6] M Ody and W Carey. 2009. Demystifying Peer Assisted Study Sessions ( PASS ): What ...? How ...? Who ...? Why ...? Chall. Learn. Dev. 6th LDHEN Symp. Bournemouth Univ. (2009), 1-9.

[7] B Singh and P Mishra. 2017. Process of Teaching and Learning: A Paradigm Shift. Int. J. Educ. 7, (June 2017), 2347-4343.

[8] V Tinto. 1975. Dropout from Higher Education: A Theoretical Synthesis of Recent Research. Rev. Educ. Res. 45, 1 (1975), 89-125. DOI:https://doi.org/10.3102/00346543045001089 